\title{
Complex Dynamics of Hydrogen Bonded Self-assembling Polymers
}

\author{
M. Wübbenhorst, J. van Turnhout \\ Department of Polymer Materials and Engineering \\ Faculty of Applied Sciences \\ Delft University of Technology \\ Delft, The Netherlands
}

\author{
B. J. B. Folmer, R. P. Sijbesma and E. W. Meijer \\ Eindhoven University of Technology \\ Laboratory of Macromolecular and Organic Chemistry \\ Eindhoven, The Netherlands
}

\begin{abstract}
Supramolecular polymers, which are non-covalently bonded and formed by self association of di or trifunctional monomers exhibit, by virtue of quadruple hydrogen bonds, many of the properties of normal high molecular weight polymers, e.g. a dynamic rubber plateau. We focus on the molecular and cooperative dynamics of self-assembled linear polymers and networks, studied by broadband dielectric relaxation spectroscopy (DRS) in the frequency range from $10^{-2}$ to $10^{6} \mathrm{~Hz}$. The DRS analysis was backed up by dynamic mechanical and rheological experiments. In the high temperature region two loss processes ( $\alpha$ and $\alpha^{*}$ ) show up, the relaxation times of which obey the Vogel-Fulcher-Tammann (VFT) law. The dielectric $\alpha$ process is related to the dynamic glass-rubber transition and is slightly faster than the corresponding mechanical $\alpha$ process. A slower (high-temperature) $\alpha^{*}$ relaxation is identified as chemical, corresponding to the mean lifetime of the hydrogen bonded linkages in the supramolecular chains. Its relaxation time $\tau_{\alpha^{*}}$ was found to be 1 to 2 decades larger than the terminal flow relaxation time, indicating that the relaxation of an entire chain is dominated by the joint dynamics of many hydrogen bonds. The $\beta$ relaxation, observed in both DRS and dynamic mechanical analysis (DMA) at temperatures below $T_{g}$, arises from the local junction dynamics of hydrogen bonded units in the glassy state. Details of the temperature dependence and the shape of the loss peaks of the $\alpha$ and $\alpha^{*}$ relaxations will be discussed in terms of temporary physical networks and cooperativity.
\end{abstract}

\section{INTRODUCTION}

$\mathrm{D}$ EVELOPMENT in the field of supramolecular chemistry [1] has revived the prospect of obtaining polymeric properties from reversible associates [2]. Supramolecular polymers consist of arrays of low molecular weight molecules linked together by directional, noncovalent interactions such as ionic, metal-coordination or hydrogen bonding. These must be stronger than the interchain van der Waals interactions in order to form linear chains selectively. Lehn and coworkers reported the first supramolecular polymer created in the liquid crystalline state in 1990 [3]. Later other groups reported the development of supramolecular polymers with liquid crystalline properties $[4,5]$. However, up to now the low molecular weight units form linear polymer chains only when they are in the liquid crystalline state.
In order to obtain self-assembling polymers with a high degree of polymerization in the amorphous state, the melt, or in dilute solutions, very strong association between the building blocks is required. Due to their strength and directionality, multiple hydrogen bond arrays have been shown to be the most promising for the development of selfassembling polymers [6,7]. Stadler in particular has been a pioneer in this area; he and his coworkers studied the properties of polybutadienes substituted with phenyl-urazole units in great detail [8-12]. However, reports on linear 'polymers' with typical polymeric properties based on reversible interactions between low molecular weight compounds, are still scarce $[13,14]$. In view of potential applications, it is a prerequisite that the compounds are easy to synthesize from cheap starting materials. Low molecular weight compounds based on 2-ureido-4 $[1 \mathrm{H}]$-pyrimidinone fulfill all these criteria. As shown in previous work [15-18], these compounds are easy accessible from inexpensive chemi- 
cals.

The physical behavior of high molecular weight macromolecules is quite different to that of low molecular weight compounds. Although both low and high molecular weight materials might undergo transitions from the liquid to the crystalline or glassy state, the existence of long macromolecules gives rise to specific slow relaxation processes involving whole polymer chains. Chain relaxation and the formation of (temporary) entanglement are therefore the reason for the complex dynamics and the viscoelastic properties of macromolecules, characterized by a sufficiently high molecular weight, a rubbery state between the amorphous glass (or semi-crystalline) and the liquid regimes.

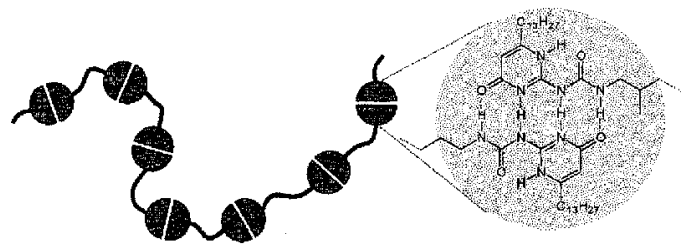

Figure 1. Schematic structure of a linear self-assembling polymer. The chemical structure of the four complementary hydrogen bonding units is given on the right.

In the present work the dynamic properties of self-assembling polymers based on quadruple hydrogen bonding ureidopyrimidinone units are addressed (Figure 1). Based on three different 'monomers', which contain one, two or three functional groups, we obtained a variety of modular, polymeric structures ranging from short linear polymers to large self-assembling networks.

The relaxation behavior of these materials was analyzed with dielectric relaxation spectroscopy as the main technique. In order to compare these results with the viscoelastic properties, in particular the slow chain relaxation and mechanical glass transition, dynamic mechanical and rheological measurements were also made.

\section{EXPERIMENTAL}

\subsection{MATERIALS AND TRANSITION CHARACTERIZATION}

The synthesis and characterization of the three H-bonding compounds shown in Figure 2 have been described [19]. Difunctional compound 1 consists of two ureidopyrimidinone units connected with a trimethylhexane spacer, and the material used is a mixture of $2,4,4-$ trimethylhexane (1a) and 2,2,4-trimethylhexane (1b) isomers. Trifunctional compound 2, which forms a self-assembling network, consists of three ureidopyrimidinone units connected by a short $\left(\mathrm{CH}_{2}\right)_{9}$ spacer.

Mixtures of 1 with $20 \% 2$, and 1 with $5 \% 3$, were obtained by dissolving the compounds in chloroform, followed by slow removal of the solvent under slightly reduced pressure at room temperature. The materials were next heated at $90^{\circ} \mathrm{C}$ under reduced pressure. Thermal transitions of all pure compounds and mixtures were determined on a Perkin Elmer Pyris 1 under a nitrogen atmosphere with heating and cooling rates of $20^{\circ} \mathrm{C} / \mathrm{min}$.

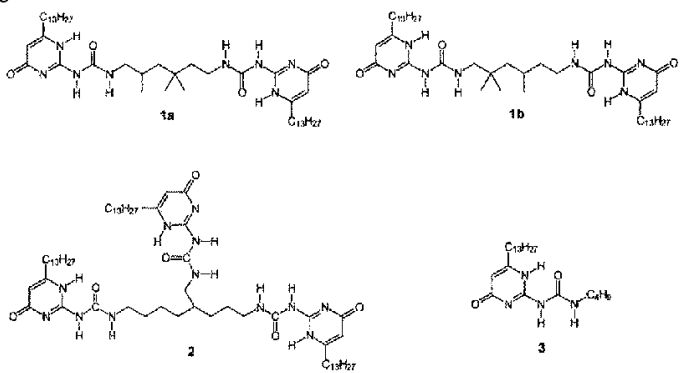

Figure 2. Chemical structures of difunctional compound 1, trifunctional compound 2 and end-capper (mono-functional) 3.

\subsection{DYNAMIC MECHANICAL THERMAL ANALYSIS}

The sub- $T_{g}$ dynamic mechanical spectra were recorded on a Perkin Elmer DMA 7 dynamic mechanical analyzer, calibrated with mercury and indium. Small, $\approx 2 \times 4 \times 20 \mathrm{~mm}^{3}$, rectangular bars of the polymer, were subjected to a sinusoidal deformation at constant frequency, using the three-point bending method. Measurements were carried out at a frequency of $1 \mathrm{~Hz}$ and a heating rate of $2^{\circ} \mathrm{C} / \mathrm{min}$. The amplitude of the sinusoidal deformation was kept the same $(25 \mu \mathrm{m})$ and the static force was always $10 \%$ more than the dynamic force in order to ensure good contact between sample and probe. The glass transition temperature, at which the samples start to creep too much, limited the upper measuring temperature.

\subsection{DYNAMIC OSCILLATORY SHEAR MEASUREMENTS}

The rheological measurements were performed on a Rheometrics RMS800 in a cone-plate geometry $(25 \mathrm{~mm})$ or a RFS II using plate-plate geometry $(50 \mathrm{~mm})$ or an ARES in a plate-plate geometry $(50 \mathrm{~mm})$. Strain amplitudes to $30 \%$ were used, which were all well within the linear viscoelastic range.

\subsection{DIELECTRIC RELAXATION SPECTROSCOPY}

The compounds were melted together with spacers (quartz fibers, diameter 200 to $500 \mu \mathrm{m}$ ) between circular brass electrodes of $20 \mathrm{~mm}$ diameter. Preparation took place either in vacuum or in a flow of nitrogen gas. The dielectric experiments were performed using a combination of two systems covering the frequency range from $10^{-2}$ to $10^{6} \mathrm{~Hz}$ : a frequency response analyzer (Schlumberger 1260) equipped with a custom made dielectric interface (developed by $\mathrm{TNO}$ ) for frequencies between $10^{-2}$ and $10^{3} \mathrm{~Hz}$, and a Hewlett-Packard $4284 \mathrm{~A}$ precision $L C R$ meter for frequencies between $10^{3}$ and $10^{6} \mathrm{~Hz}$. The sample was placed in a nitrogen cryostat (Novocontrol), the temperature of which was controlled with a stability of better than $\pm 50 \mathrm{mK}$. 


\section{RESULTS AND DISCUSSION}

\subsection{RELAXATION PROCESSES IN LINEAR SELF-ASSEMBLING POLYMERS}

An overview of the dielectric relaxation processes in compound 1 is given in Figure 3. Three relaxation processes, labeled by the symbols $\alpha, \beta$ and $\gamma$, can be recognized readily, whereas a 4 th relaxation $\left(\alpha^{*}\right)$ appears as a shoulder at temperatures above the $\alpha$ relaxation. The most prominent relaxation is the $\alpha$ transition, which can be identified with the dynamic glass transition involving segmental motion of parts of the supramolecular chain. The very existence of such a relaxation process, the assignment of which was confirmed by calorimetric results, clearly demonstrates the behavior of compound $\mathbf{1}$ as a genuine polymer. At lower temperatures, or at higher frequencies, a weaker $\beta$ relaxation can be seen, which should be related to local dynamics in the glassy state. A corresponding relaxation process also has been found in DMA measurements, where at $-60^{\circ} \mathrm{C}$ a maximum emerged in the loss modulus. Furthermore, at very low temperature, a $\gamma$ relaxation shows up.

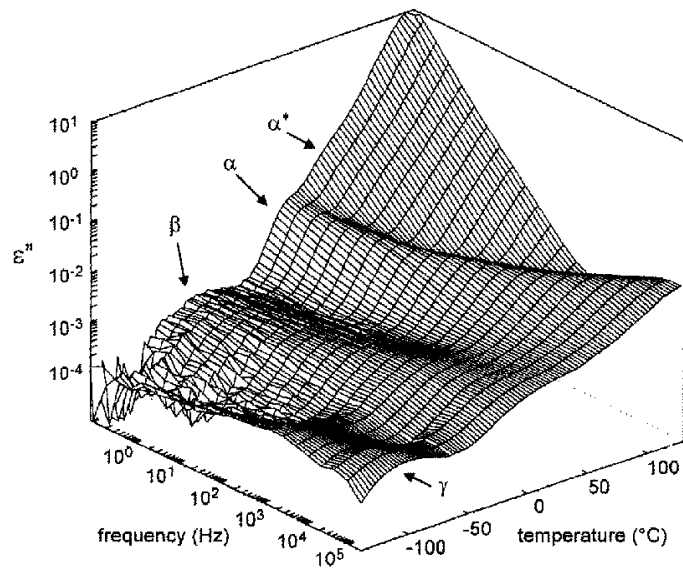

Figure 3. 3D representation of the dielectric loss $\varepsilon^{\prime \prime}$ of 1 as a function of frequency and temperature.

The fourth, $\alpha^{*}$, process can be noticed only marginally in the dielectric $\operatorname{loss} \varepsilon^{\prime \prime}$, because ohmic conduction dominates the dielectric loss at temperatures above the $\alpha$ process. In order to eliminate conduction from the dielectric loss curves, we have calculated the alternative loss $\varepsilon_{d}^{\prime \prime}$ from the permittivity $\varepsilon^{\prime}$, which has proven to be successful in previous work $[20,21]$, according to

$$
\varepsilon_{d}^{\prime \prime}=-\frac{\pi}{2} \frac{\partial \varepsilon^{\prime}(\omega)}{\partial \ln (\omega)} \approx \varepsilon^{\prime \prime}
$$

This yields a fair approximation of the 'conduction free' loss $\varepsilon^{\prime \prime}$ for broad relaxation peaks, while as a bonus for narrow Debye-like relaxation peaks, an additional sharpening is achieved [20]. The effectiveness of this procedure is illustrated for compound 1 in Figure 4.

An essential basis for the assignment of the different relaxation processes to specific molecular motions, is the temperature dependence of their mean relaxation time $\tau$, which in most cases either obeys an
Arrhenius law

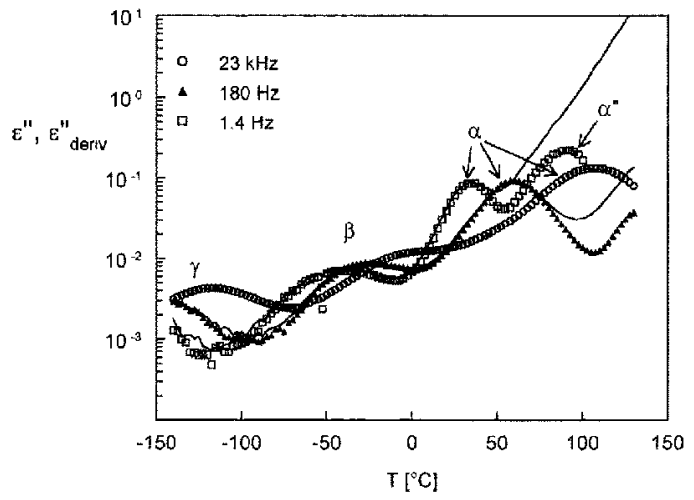

Figure 4. $\varepsilon_{d}^{\prime \prime} v$ s. temperature of compound 1 , at three different frequencies $f$, the four observed relaxations are denoted with $\alpha, \alpha^{*}, \beta$ and $\gamma$. For comparison, the measured loss $\varepsilon^{\prime \prime}(T)$ at the two lowest frequencies are shown as lines as well.

Table 1. Activation parameters for the different relaxation processes of 1 according to a (1) Arrhenius equation (Equation 2) or (2) VFT equation (Equation 3) relaxation.

\begin{tabular}{c|c|c|c|c|}
\hline Relaxation & $\begin{array}{c}\log \tau_{\infty} \\
\mathrm{s}\end{array}$ & $\begin{array}{c}E_{a} \\
\mathrm{~kJ} / \mathrm{mol}\end{array}$ & $\begin{array}{c}E_{V} \\
\mathrm{~kJ} / \mathrm{mol}\end{array}$ & $\begin{array}{c}T_{V} \\
\mathrm{~K}\end{array}$ \\
\hline$\alpha^{*}$ & -5.35 & - & 14.9 & 221 \\
$\alpha$ & -10.4 & - & 14.9 & 221 \\
$\beta$ & -19.5 & 75.4 & - & - \\
$\gamma$ & -12.0 & 20.8 & - & - \\
\hline
\end{tabular}

or, in case of cooperative segmental $(\alpha)$ relaxations, the Vogel-FulcherTammann (VFT) equation

$$
\tau(T)=\tau_{\infty} \exp \left[\frac{E_{V}}{R\left(T-T_{V}\right)}\right]
$$

Here the parameters $R, E_{a}, E_{V}, T_{V}$ and $\tau_{\infty}$ denote the gas constant, the activation energy, the 'Vogel activation energy', the 'Vogel scaling temperature' and the pre-exponential factor. In order to determine the mean relaxation time $\tau(T)$ from the dielectric loss curves, we have fitted the frequency spectra $\varepsilon^{\prime \prime}(\omega)$ with a set of two HavriliakNegami (HN) relaxation functions (Equation (4) by using the LevenbergMarquardt algorithm

$$
\varepsilon^{\prime \prime}=-\sum_{k=1}^{2} \mathfrak{I}\left\{\frac{\Delta \varepsilon_{k}}{\left[1+\left(i \omega \tau_{k}\right)^{a_{k}}\right]^{b_{k}}}\right\}+\frac{\sigma}{\varepsilon_{o} \omega}
$$

where $\Delta \varepsilon_{k}$ and $\tau_{k}$ correspond to the relaxation strength and the mean relaxation time of the $k$ th process. The two shape parameters $a_{k}$ and $b_{k}$, which determine the slope $d \ln \varepsilon^{\prime \prime} / d \ln \omega$ of the low frequency loss tail $a$ and the high frequency loss tail $-a b$, are determined by the underlying distribution in relaxation times. The 2nd term in Equation (4) accounts for the pure ohmic conduction $\sigma$.

The relaxation times obtained by the HN fit procedure are presented in a relaxation map (Figure 5) and portray two VFT and two Arrhenius dependencies. The VFT and Arrhenius parameters are summarized in Table 1. 


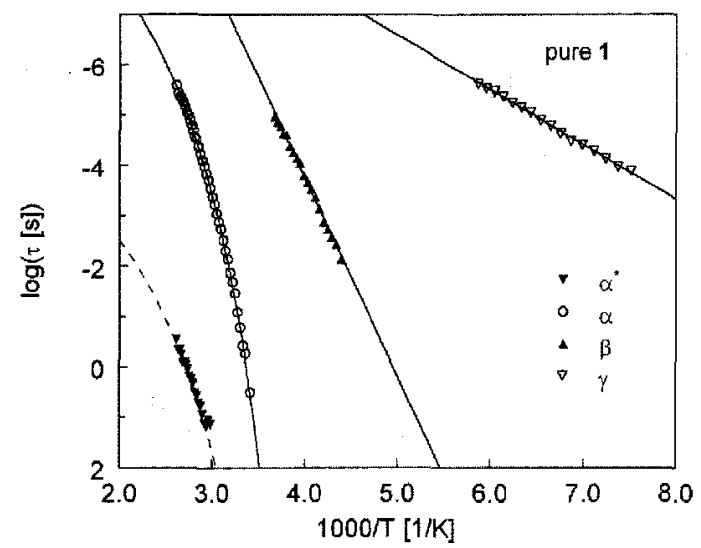

Figure 5. Arrhenius plot $(\log (\tau)$ vs. $1 / T)$ of the four dielectric relaxation processes of 1 .

One clearly sees that the $\alpha$ relaxation displays the curvature according to the VFT law as a characteristic feature of the dynamic glass transition, which is supported by a fair agreement of the glass transition temperatures found by differential scanning calorimeter (DSC) $\left(26.8^{\circ} \mathrm{C}\right)$ and dielectric relaxation spectroscopy (DRS) $\left(21^{\circ} \mathrm{C}\right.$ ).

By contrast, the $\beta$ relaxation shows a clear Arrhenius behavior, but its high activation energy and extremely small pre-exponential factor $\left(\log \tau_{\infty}=-19.5\right)$ imply that the $\beta$ process is not a simple thermally activated relaxation, but invokes entropy effects as well. Most likely, the $\beta$ relaxation reflects the dynamics of bending fluctuations of the associated hydrogen bonded units in combination with fast dissociation and re-association of the hydrogen bonded units in the glassy state.

The $\gamma$ process on the other hand, is a very low thermally activated process which shows typical Arrhenius features. This relaxation occurs at a low temperature and high frequency, indicating that a very local process is involved, which points to a rotation of the alkyl side chains.

The molecular origin of the $\alpha^{*}$ relaxation is ruled by the dynamics of the hydrogen bonded units, yet it follows closely the relaxation time of the $\alpha$ process, i.e. it obeys the VFT-law. Although both processes are clearly interrelated, the relaxation time of the $\alpha^{*}$ process is $\sim 5$ decades longer than that of the $\alpha$ relaxation. Furthermore, the shape parameters $a$ and $b$ in the Havriliak-Negami fit (Equation (4)) are found to be near one, which means that the process exhibits a Debye-like behavior, which in turn implies a single relaxation time. We will discuss this relaxation process further in Section 3.3 .

\subsection{CROSSLINKED AND TERMINATED SELF-ASSEMBLING POLYMERS}

A straightforward approach to verify the above assignments of the relaxation processes in compound 1 is a systematic variation of the selfassembling, polymeric chain structure. Based on the statistics for polymerization reactions for conventional macromolecules, developed by Flory, the degree of polymerization, crosslink density, or other structure parameters of supramolecular polymers, can be tuned easily just by adding an endcap (which provokes monofunctionality and thus terminates the linear supramolecular chain) or by adding a trifunctional rather than a bi-functional compound.

It has been shown that the addition of even $<5 \%$ of a mono-functional compound (3) to a solution of difunctional compounds in chloroform, resulted in a dramatic drop in viscosity, indicating that the degree of polymerization of the reversible polymer had decreased. On the other hand, the addition of a trifunctional compound (2) to a solution of a difunctional compound in chloroform resulted in a marked increase in the solution's viscosity, which in turn indicates that the degree of polymerization had increased. The bulk properties of mixtures of difunctional compound 1 with an amount of mono-functional compound 3 or trifunctional compound 2, have been determined by DSC, DRS and rheology [19] (Table 2).

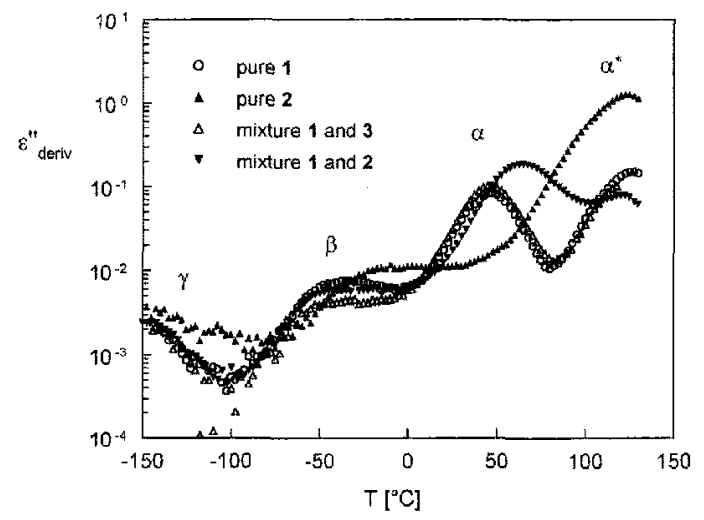

Figure 6. 'Conduction free' loss $\varepsilon_{d}^{\prime \prime}$ vs. temperature of compounds 1 , 3 and binary mixtures $1+2$ and $1+3$, respectively.

As for compound 1 (see Section 2.1), we have performed a DRS analysis on three types of samples: 100\% trifunctional compound 2, a mixture of $20 \% 2$ (trifunctional) and difunctional material 1 and a mixture of $5 \% 3$ (mono-functional) and 1 . A comparison of the dielectric loss $\varepsilon_{d}^{\prime \prime}$ at $f=22 \mathrm{~Hz}$ for all materials is shown in Figure 6 .

The dielectric loss curve of the trifunctional compound 2 exhibits the largest changes with respect to compound 1 i it displays at first sight only three dielectric relaxation processes $(\alpha, \beta$, and $\gamma)$, from which the $\gamma$ relaxation coincides more or less with that found for compound 1 . By contrast, the $\beta$ relaxation is significantly shifted towards higher temperatures, which corresponds to a delay in the local sticker dynamics by more than one decade ( $c f$. Figure 7). This delay can be rationalized by structural differences in the central flexible part in compounds 1 and 3 , which links the stiff $\mathrm{H}$-bonding units and provides for most of the polymer chain flexibility. Obviously, the presence of the third ureidopyrimidinone group in 2 causes less flexibility and increases steric hindrance in the alkyl link. From the Arrhenius parameters in Table 3 we further note that the delay in relaxation time is caused predominantly by an increase in the pre-exponential factor, while the activation energy of the dielectric $\beta$ process remains almost unchanged $(75.4 \mathrm{~kJ} / \mathrm{mol}$ (1) $\rightarrow 71.4 \mathrm{~kJ} / \mathrm{mol}$ (2). Since these values coincide neatly with the activation energies found from exchange NMR experiments [22] on monofunctional model compounds in solution, we must conclude that the 
Table 2. Glass transition temperatures determined by DSC and DRS for all compounds polymer. * From an activation energy fine-structure analysis at $f=90 \mathrm{~Hz}$

\begin{tabular}{|c|r|r|r|}
\hline Polymer & $\begin{array}{r}T_{g} \\
{ }^{\circ} \mathrm{C}\end{array}$ & $\begin{array}{c}T_{g}\left(E_{a}\right) \\
{ }^{\circ} \mathrm{C}\end{array}$ & $\begin{array}{c}E_{a}(\max ) \\
\mathrm{kJ} / \mathrm{mol}\end{array}$ \\
\hline 1 & 21 & 26.8 & 151 \\
2 & 72 & 69.7 & 293 \\
$1+5 \% 3$ & $<20$ & 17.7 & 220 \\
$1+20 \% 2$ & 33 & 30.3 & 244 \\
\hline
\end{tabular}

Table 3. Activation parameters for the $\gamma$ and $\beta$ dielectric relaxation of polymers 1 and 2 .

\begin{tabular}{|c|c|c|c|}
\hline Compound & Type & $\log \tau_{\infty}$ & $\begin{array}{c}E_{a} \\
\mathrm{~s} / / \mathrm{mol}\end{array}$ \\
\hline 1 & $\gamma$ & -12.0 & 20.8 \\
1 & $\beta$ & -19.5 & 75.4 \\
2 & $\beta$ & -17.2 & 71.4 \\
\hline
\end{tabular}

energy barrier of the $\beta$ relaxation reflects the fast, reversible, distortion caused by cooperative breaking and reformation of a set of quadruple $\mathrm{H}$ bonds in the glassy state.

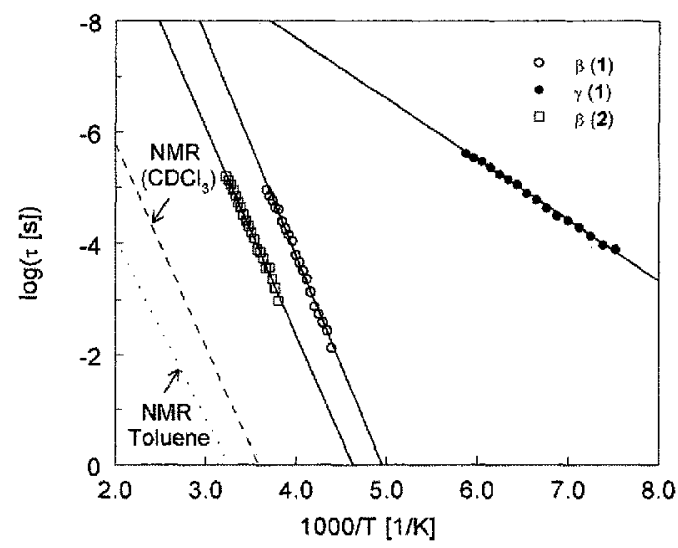

Figure 7. Arrhenius plot of the secondary relaxations in 1 and 2. As a reference, relaxation times from NMR are depicted also. $\mathrm{CDCl}_{3}: E_{a}=70 \mathrm{~kJ} / \mathrm{mol}_{r} \log \tau_{\infty}=-13.1$, toluene-d $\mathrm{d}_{8}: E_{a}=62 \mathrm{~kJ} / \mathrm{mol}_{\text {, }}$ $\log \tau_{\infty}=-10.6$.

Another manifestation of the reduced flexibility of the polymer chain due to the increase in functionality from 2 in case of the linear polymers to 3 in case of self-assembling networks, is the large upshift of the $\alpha$ relaxation. The $T_{g}$ of 2 , calculated form $\varepsilon^{\prime}(f, T)$ by the activation energy fine structure analysis according to van Turnhout et al. [23, 24], was found to be $70^{\circ} \mathrm{C}$, which is in fair agreement with the value obtained with DSC measurements $\left(72^{\circ} \mathrm{C}\right)$. Although a separate $\alpha^{*}$ process seems to be absent, closer analysis of the high-temperature relaxation does reveal the existence of two narrowly distributed relaxation processes, with relaxation times that are separated by $<1$ decade.

As expected, the mixture of a crosslinking (2) and bi-functional com-' pound 1 results in a glass transition temperature in between those of compounds 1 and 2 . This is indicated by a significant shift in the $\alpha_{\text {DRs }}$ peak to higher temperatures or lower frequencies. Furthermore, the shape of the $\alpha$ peak of the mixture differs significantly from that of pure

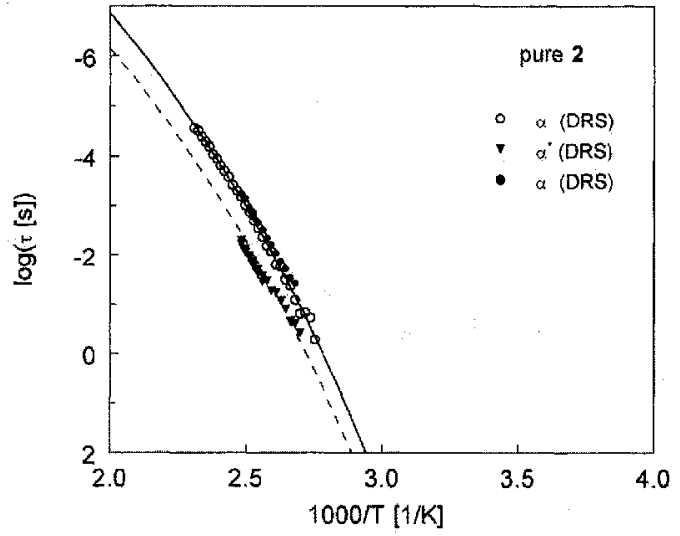

Figure 8. Arrhenius plot $(\log (\tau)$ vs, $1 / T)$ of the dielectric $\alpha$ and $\alpha^{*}$ process of 2 .

1: The decreased slope at low frequencies $(a=0.7)$ (detectable as a flat high temperature part of the $\alpha$-peak in Figure 6), when interpreted in terms of increased intermolecular cooperativity of the segmental motion [25], is a strong hint for the presence of physical crosslinks in the material. The other relaxations, $\beta, \gamma$ and $\alpha^{*}$, show only marginal changes with respect to material 1 .

Moreover, the dielectric spectra of the second mixture, $1+20 \% 3$, show four relaxations $\left(\alpha, \beta, \gamma\right.$, and $\left.\alpha^{*}\right)$. By comparing the observed relaxation processes of the mixture with those of pure 1 it becomes immediately clear that addition of monofunctional compound 3 to polymer 1 , does not result in a shift in the $\alpha$ and $\alpha^{*}$ relaxations (Figure 6).

\subsection{ANALOGY BETWEEN MECHANICAL AND DIELECTRIC PROCESSES}

Let us now compare the dielectric relaxation behavior with results obtained from dynamic mechanical measurements. For this purpose we have displayed the temperature dependence of the dielectric $\left(\varepsilon^{\prime}\right.$ and $\left.\varepsilon^{\prime \prime}\right)$ and dynamic mechanical moduli $\left(E^{\prime}\right.$ and $\left.E^{\prime \prime}\right)$ of the mixture $1+$ $20 \% 2$ in the same graph (Figure 9).

At first sight, there is close similarity between the number and the position of the loss peaks in $E^{\prime \prime}(T)$ and $\varepsilon^{\prime \prime}(T)$ near $1 \mathrm{~Hz}$. The $\beta$ relaxation, which shows up as the first relaxation process in the DMA curve at low temperatures, appears at the same peak temperature as the dielectric $\beta$ peak, implying the same molecular mechanism for the mechanical and dielectric process. Similar peak positions are found also for the dielectric $\alpha$ process $\left(\alpha_{\mathrm{DRS}}\right.$ ) and the corresponding mechanical $\alpha_{\mathrm{DMA}}$ relaxation, since both are related to the dynamic glass transition. The remaining differences in the dielectric and mechanical $\alpha$ peak location might arise from experimental uncertainties in the DMA measurements close to the glass transition temperature.

Additional data about the mechanical $\alpha$ relaxation were collected from rheological experiments in the frequency domain [19]. The relaxation times of the $\alpha_{\mathrm{DMA}}$ process are given together with those of the dielectric $\alpha$ and $\alpha^{*}$ processes for compound 1 and mixture $1+20 \% 2$ in 


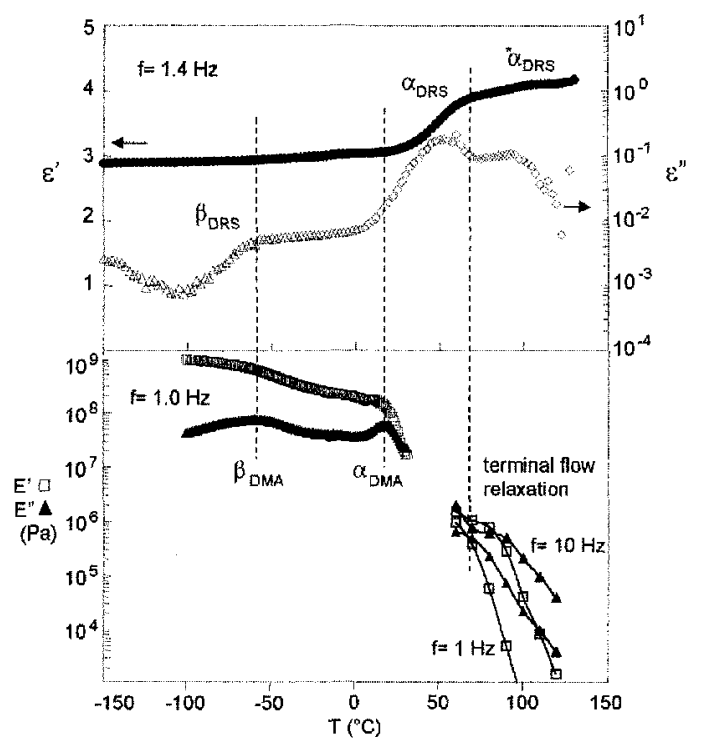

Figure 9. Temperature dependence of the dielectric $\left(\varepsilon^{\prime}\right.$ and $\left.\varepsilon^{\prime \prime}\right)$ and dynamic mechanical moduli ( $E^{\prime}$ and $E^{\prime \prime}$ ) of the mixture.

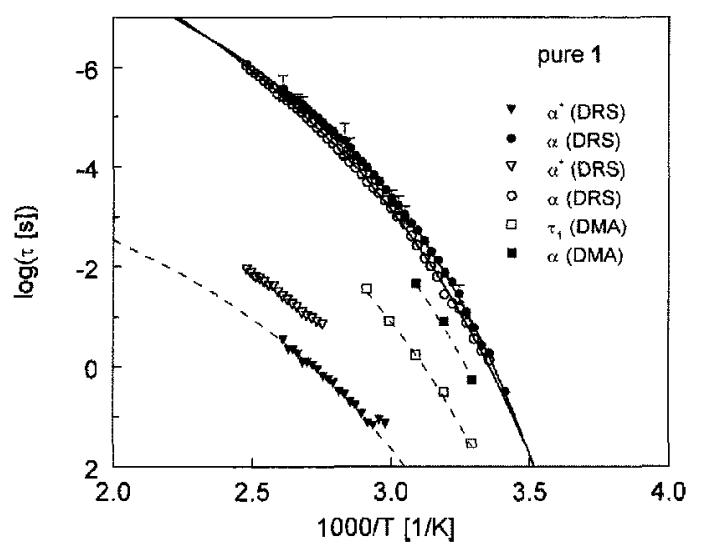

Figure 10. Relaxation map of all dielectric and mechanical relaxation times encountered in difunctional compound 1. Dielectric data from two samples exposed to different maximum temperatures are presented. (a) filled symbols $T_{\text {max }}=130^{\circ} \mathrm{C}$, open symbols refer to $T_{\max }=110^{\circ} \mathrm{C}$. Solid lines are obtained by VFT fit of the $\alpha$ process. The dashed lines are based on the same VFT parameters $E_{V}$ and $T_{V}$, and were shifted vertically by adjusting $\tau_{\infty \mathrm{s}}$.

Figures 10 and 11. As expected, the dielectric and mechanical relaxation times again were found to be similar within one decade in frequency.

The analogy between the dielectric and mechanical behavior, which holds for both the $\beta$ and $\alpha$ relaxation (Figure 6), obviously fails above the glass transition region. Although there is a gap between the mechanical data measured by DMA and the theological experiment due to instrumental constrains, one clearly recognizes the dynamic rubber plateau in $E^{\prime}$ followed by the terminal flow region as indicative for (macromolecular) chain dynamics. The transition from the rubber plateau to viscous flow usually is quantified by the terminal flow relax-

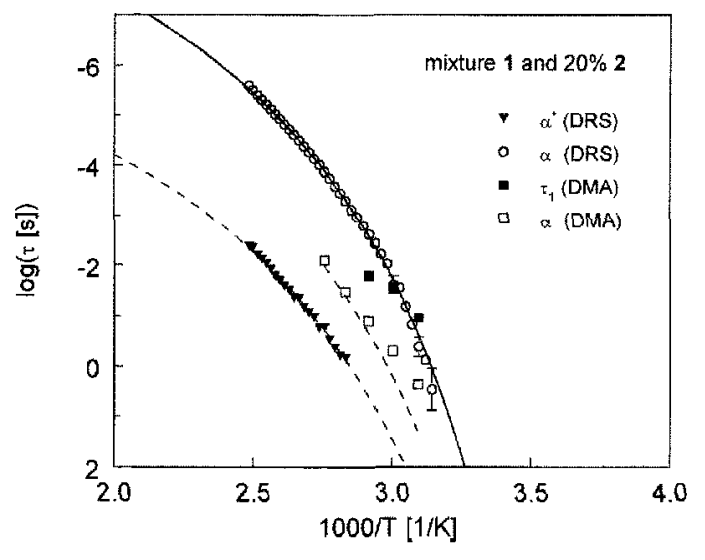

Figure 11. Arrhenius plot of the mean relaxation time of the $\alpha$ and $\alpha^{*}$ relaxation as observed with DRS and the terminal flow relaxation time as observed in DMA vs. the reciprocal temperature of a mixture of 1 with $20 \% 2$

ation time $\tau_{1}$ which corresponds to the relaxation time of the polymer along its critical path (or end-to-end vector) in the entangled (reptation model) or non-entangled (Rouse model) regime. $\tau_{1}$ relaxation times for 1 and mixture $1+20 \% 2$ were determined from isothermal frequency sweeps $G^{*}(\omega)$ and are shown in Figures 9 and 10 . The relaxation times for the dielectric $\alpha^{*}$ process are displayed in the same graphs.

Let us first focus on Figure 10, which compiles all mechanical and dielectric relaxation times of the difunctional compound 1. As already suggested by the occurrence of a rubber plateau, the terminal flow relaxation time is $\sim 2$ decades longer than the characteristic time for segmental motion, represented by the dielectric or mechanical $\alpha$ process. The most striking result is that the relaxation time $\tau$ of the $\alpha^{*}$ process is again $>2$ orders of magnitude slower than the terminal flow relaxation time.

The finding that the dielectric $\alpha^{*}$ relaxation is the slowest relaxation process in the reversible polymer 1 , supports its assignment to a 'chemical relaxation', i.e. a process which reflects the mean lifetime of the hydrogen bonded linkages. Such relaxations have been discussed by e.g. Müller and co-workers $[9,11]$ who studied the junction dynamics of thermoreversible networks. In their work they showed that both dissociation/association, the true chemical relaxation, and reorientation of closed $\mathrm{H}$ stickers may contribute to a slow $\alpha^{*}$ relaxation process.

In our case of quadruple $\mathrm{H}$-bonding units, only open $\mathrm{H}$ bonds contribute to the $\alpha^{*}$ relaxation, since a closed bond has no dipole moment. Since the reorientation dynamics of open stickers occurs at the time scale of segmental motion and therefore likely contributes to the dielectric $\alpha$ process, the $\alpha^{*}$ relaxation should manifest the rate of both dissociation and association of the ensemble of $\mathrm{H}$-bonded units. Considering the high dimeric association constants found by NMR and excimer fluorescent experiments [22], we know that the dissociation rate is several orders of magnitude faster than the association (recombination) process. Consequently, the relaxation time $\tau_{\alpha *}$ clearly represents the mean lifetime of closed linkages rather than the lifetime of free H-bonding units. This is corroborated by two other features of the dielectric $\alpha^{*}$ relaxation, its narrow distribution of relaxation times (Debye-like pro- 
cess) and its admittedly low dielectric relaxation strength. A quantitative analysis and molecular modeling of the $\alpha^{*}$ relaxation processes will be subject of a forthcoming paper.

The identification of $\tau_{\alpha *}$ as the lifetime of individual $\mathrm{H}$ bonds immediately answers the question why the terminal flow relaxation time was found to be much shorter than $\tau_{\alpha *}$. It is obvious that the mean lifetime' of a whole polymer chain, which consists of $N$ dimeric contacts, is proportional to $N \tau_{\alpha^{*}}$. Whereas for conventional entangled polymers reptation is the most important mechanism of stress relaxation [26-30], self-assembling polymers can relax by an extra pathway, e.g. by the dissociation and association of the hydrogen bonds. The viscoelastic behavior of reversible, breakable polymer chains has been described theoretically by Cates [31,32]. He showed that when the lifetime of the hydrogen bond units $\left(\tau_{b}\right)$ is much larger than the characteristic lifetime of entanglement $\left(\tau_{r}\right)$, the reversible polymer will show stress relaxation with a relaxation lifetime equal to the lifetime of entanglement.

Conversely, when $\tau_{b}$ is similar or shorter than the lifetime of the entanglement, still a sharp transition from the rubber plateau to the terminal flow region might be observed, the relaxation time $\tau_{1}$ of which equals according to Equation (5)

$$
\tau_{1}=\left(\tau_{b} \tau_{r}\right)^{\frac{1}{2}}
$$

In other words, the occurrence and width of the rubber plateau is controlled by the dissociation and association of the hydrogen bonds.

We want to check this molecular view by considering the results for the other materials, which relaxation maps are presented in Figures 8 and 11 , respectively.

As already noticed, the trifunctional compound 2 shows an $\alpha^{*}$ relaxation time very close to that of the $\alpha$ process. Since the chain relaxation time $\tau_{1}$ cannot exceed the individual lifetime of the relaxing dynamic unit, the terminal flow relaxation is expected to occur at a time scale similar to that of the segmental motions. This is confirmed by the rheological results of 2 , which material shows no rubber plateau.

As expected, the mixture of 1 with $20 \%$ trifunctional 2 portrays properties in between the pure difunctional and pure trifunctional materials. The rubber plateau is found to be less pronounced than in $\mathbf{1}$, which is accompanied by a lower ratio of $\tau_{\alpha^{*}}$ and $\tau_{1}$. This again implies that the chain relaxation time is controlled by the lifetime of the individual hydrogen bonds reflected by $\tau_{\alpha^{*}}$.

Whereas dissociation and association of the hydrogen bonds is most likely the limiting factor for the chain relaxation time $\tau_{1}$ in the new materials discussed above, the mixture containing end-caps $(1+5 \% 3)$ represents the case, where the (short) lifetime of entanglement $\left(\tau_{\text {rep }}\right)$ dictates $\tau_{1}$. The presence of end-caps causes a strong decrease in the virtual molecular weight, which in turn results in the absence of a rubber plateau in the master curves of $G^{\prime}$ and $G^{\prime \prime}$ [19] due to shortening of the terminal flow relaxation time towards $\tau_{\alpha}$. Surprisingly, this does not affect the lifetime of dimeric stickers, indicated by $\tau_{\alpha^{*}}$.

\section{CONCLUSIONS}

$\mathrm{T}$ HE complex dynamics of new self-assembling linear polymers and networks, based on quadruple hydrogen bonding units, has been studied by broadband dielectric relaxation spectroscopy (DRS), dynamic mechanical analysis and rheology. Four dielectric relaxation processes were revealed and assigned to molecular motions and to the dynamics of breaking and remaking of $\mathrm{H}$-bonds in the glassy and rubbery state.

The dielectric $\alpha$ process is related to the dynamic glass-rubber transition and is slightly faster than the corresponding mechanical $\alpha$ process. The slowest (high-temperature) dielectric $\alpha^{*}$ relaxation was identified as a chemical relaxation, which is related to the mean lifetime of the hydrogen bonded linkages in the supramolecular chains. Its relaxation time $\tau_{\alpha^{*}}$ was found to be 1 to 2 decades larger than the terminal flow relaxation time $\tau_{1}$, which indicates that the self-assembled polymer chains consist of a large number of hydrogen bonded units.

The $\beta$ relaxation, observed in both DRS and DMA at temperatures below $T_{g}$, was assigned to the local dynamics of hydrogen bonded units in the glassy state, which most likely involves the fast breaking and reformation of $\mathrm{H}$ bonds in a cooperative way. Our study has demonstrated that self-assembling polymers based on very strong hydrogen bonding exhibit relaxation processes typical of normal polymers and consequently have bulk properties usually only attributed to high molecular weight covalently bonded polymers.

\section{ACKNOWLEDGMENT}

The authors like to thank B. Norder and J. P. W. Peters for performing the mechanical and the rheological measurements.

\section{REFERENCES}

[1] J.-M. Lehn, Molecular Chemistry, Weinheim: VCH, 1995.

[2] N. Zimmerman, J.S. Moore, and S. C. Zimmerman, "Polymer Chemistry comes full Circle", Chem. Ind., pp. 604-610, 1998.

[3] C. Fouquey, J.-M. Lehn, and A.-M. Levelut, "Molecular Recognition Directed SelfAssembly of Supermolecular Liquid Crystalline Polymers From Complementary Components", Adv. Mater., Vol. 2, pp. 254-257, 1990.

[4] T. Kato and J.-M. Fréchet, "Stabilisation of a Liquid-Crystalline Phase Through Noncovalent Interaction with a Polymer Side Chain", Macromolecules, Vol. 22, pp. 3818-3819, 1989.

[5] A. Ciferri, "The Assembling Power of the Supramolecular Liquid Crystal", Trends Polym. Sci., Vol. 5, pp. 142-146, 1997.

[6] L. M. Wilson, "Structure of a Hydrogen-Bonded Liquid-Crystalline Network", Macromolecules, Vol. 27, pp. 6683-6686, 1994.

[7] C. B. St Pourcain and A. C. Griffin, "Thermoreversible Supramolecular Network with Polymeric Properties", Macromolecules, Vol, 28, pp. 4116-4121, 1995.

[8] U. Seidel, R. Stadler and G. G. Fuller, "Relaxation Dynamics of Bidisperse Temporary Networks", Macromolecules, Vol. 27, pp. 2066-2072, 1994.

[9] M. Muller, F. Kremer, R. Stadler, E. W. Fischer and U. Seidel, "The MolecularDynamics of Thermoreversible Networks as Studied by Broad-Band DielectricSpectroscopy", Colloid and Polymer Science, Vol. 273, pp. 38-46, 1995.

[10] M. Muller, U. Seidel and R. Stadler, "Influence of Hydrogen-Bonding on the Viscoelastic Properties of Thermoreversible Networks - Analysis of the Local Complex Dynamics", Polymer, Vol. 36, pp. 3143-3150, 1995.

[11] M. Muller, R. Stadler, F. Kremer and G. Williams, "On the Motional Coupling between Chain and Junction Dynamics in Thermoreversible Networks", Macromolecules, Vol. 28, pp. 6942-6949, 1995.

[12] C. I. D. Bica, W. Burchard, and R. Stadler, "Behavior of Modified and Unmodified Polybutadienes in the Semidilute Regime", Eur. Polym. J., Vol. 33, pp. 1759-1766, 1997.

[13] J. Hirschberg, F. H. Beijer, H. A. van Aert, P. Magusim, R. P. Sijbesma and E. W. Meijer, "Supramolecular Polymers from Linear Telechelic Siloxanes with Quadruple -Hydrogen-Bonded Units", Macromolecules, Vol. 32, pp. 2696-2705, 1999.

[14] R. K. Castellano, D. M. Rudkevich, and J. Rebek, Jr., "Polycap: Reversibly Formed Polymeric Capsules", Proc. Natl. Acad. Sci. USA, Vol. 94, pp. 7132-7137, 1997. 
[15] R. P. Sijbesma, F. H. Beijer L. Brunsveld, B. J. B. Folmer, J. H. K. Hirschberg, R. F. M. Lange, J. K. L. Lowe, and E. W. Meijer, "Reversible Polymers Formed from SelfComplementary Monomers Using Quadruple Hydrogen Bonding", Science, Vol. 278, pp. 1601-1604, 1997.

[16] F. H. Beijer, Cooperative multiple hydrogen bonding in supramolecular chemistry, $\mathrm{PhD}$ Thesis, Eindhoven University of Technology, May 1998.

[17] F. H. Beijer, R. P. Sijbesma, H. Kooijman, A. L. Spek, and E. W. Meijer, "Strong Dimerization of Ureidopyrimidones via Quadruple Hydrogen Bonding", J. Am. Chem. Soc., Vol. 120, pp. 6761-6769, 1998.

[18] B. J. B. Folmer, R. P. Sijbesma, and E. W. Meijer, "Polymeric Materials Based on Reversible Supramolecular Assemblies", PMSE, Vol. 217, pp. 039-PMSE, 1999.

[19] B. J. B. Folmer, "New Polymers Based on the Quadruple Hydrogen Bonding Motif", Eindhoven University of Technology, pp. 141, 2000.

[20] M. Wübbenhorst, E. Van Koten, J. Jansen, M. Mijs and J. van Turnhout, "Dielectric Relaxation Spectroscopy of Amorphous and Liquid Crystalline Side Chain Polycarbonates", Macromol. Rapid Commun., Vol. 18, pp. 139-147, 1997.

[21] I. J. A. Mertens, M. W bbenhorst, W. D. Oosterbaan, L. W. Jenneskens and I. van Turnhout, "Novel Polymer Electrolytes Based on Amorphous Poly (ether-ester)s Containing 1, 4, 7-Trioxanonyl Main Chain Units. Ionic-Conductivity versus Polymer Chain Mobility", Macromolecules, Vol. 32, pp. 3314-3324, 1999.

[22] S. H. M. Söntjens, R. P. Sijbesma, M. H. P. van Genderen and E. W. Meijer, "Stability and Lifetime of Quadruply Hydrogen Bonded 2-Ureido-4 [1H]-pyrimidinone Dimers" , J. Am. Chem. Soc, Vol. 122, pp. 7487-7493, 2000.

[23] J. van Turnhout, "New Methods for the Measurement and Analysis of Dielectric Relaxations in Polymers", presented at 15th Europhys. Conf. on Macromolecular Physics, Hamburg, EPS, Genève, 1983.
[24] P. A. M. Steeman and J. van Turnhout, "Fine-Structure in the Parameters of Dielectric and Viscoelastic Relaxations", Macromolecules, Vol. 27, pp. 5421-5427, 1994.

[25] R. Böhmer, K. L. Ngai, C. A. Angell, and D. J. Plazek, "Nonexponential Relaxations in Strong and Fragile Glass Formers", J. Chem. Phys., Vol. 99, pp. 4201-4209, 1993.

[26] P. G. de Gennes, Scaling Concepts in Polymer Physics, Ithaca Cornell University Press, 1979.

[27] M. Doi and S. F. Edwards, "Dynamics of Concentrated Polymer Systems. I. Brownian Motion in the Equilibrium State", J. Chem. Soc. Faraday Trans. II, Vol. 74, pp. $1789-1801,1978$.

[28] M. Doi and S. F. Edwards, "Dynamics of Concentrated Polymer Systems. II. Molecular Motion under Flow", J. Chem. Soc. Faraday Trans. İ, Vol. 74, pp. 1802-1817, 1978.

[29] M. Doi and S. F. Edwards, "Dynamics of Concentrated Polymer Systems. III. The Constitutive Equation"', J. Chem. Soc. Faraday Trans. II, Vol. 74, pp. 1802-1832, 1978.

[30] M. Doi and 5. F. Edwards, "Dynamics of Concentrated Polymer Systems. IV. Rheological Properties", J. Chem. Soc. Faraday Trans. II, Vol. 75, pp. 38-54, 1979.

[31] M. E. Cates, "Nonlinear Viscoelasticity of Wormlike Micelles and Other Reversibly Breakable Polymers", J. Phys. Chem., Vol. 94, pp. 371-375, 1990.

[32] M. E. Cates, "Reptation of Living Polymers: dynamics of Entangled Polymers in the Presence of Reversible Chain-Scission Reactions", Macromolecules, Vol. 20, pp. 2289-2296, 1987.

This paper is based on a presentation given at the 6th International Conference on Dielectrics and Related Phenomena, Spała, Poland, 6-10 September 2000.

Manuscript was received on 6 September 2000, in final form 6 February 2001. 